\title{
Condiciones de vida de la infancia y adolescencia afectada por eventos climáticos del 2010 en La Mojana Sucreña
}

\author{
Rubiela Godín Díaz', Blanca Pérez Contreras², Elcie Tamara Arrazola ${ }^{3}$
}

\section{RESUMEN}

El artículo presenta los resultados de la reflexión en torno a condiciones de vida de la infancia y adolescencia afectada por la ola invernal del 2010 en la subregión Mojana, en el marco del componente "Protocolo de atención a la infancia y adolescencia afectada por cambios climáticos en el Departamento de Sucre", del proyecto Inbiomédicas ejecutado en cooperación con CECAR, Gobernación y Sistema General de Regalías. Para el diseño del protocolo se requirió conocer el estado de los derechos de infantes y adolescentes en lo pertinente a la salud, hábitat, educación y participación, lo que develó las condiciones de vida de éste grupo etario. Los desastres naturales son escenarios degradantes en tanto generan desestabilización en la economía familiar, pérdidas de bienes inmuebles, afectaciones en la salud, educación, seguridad alimentaria y la dinámica familiar de cualquier población. En particular, la infancia y adolescencia es la más susceptible de ser impactada negativamente por el fenómeno, dadas sus condiciones de indefensión, no obstante, se ha invisibilizado su situación, los informes oficiales son generalistas. El enfoque de investigación fue de complementariedad basado en el método de análisis cuantitativo y cualitativo. Se trabajó

1 CECAR. Colombia. Correo electrónico: rubiela.godin@cecar.edu.co. Magíster en Estudios de Familia. Trabajadora Social. Docente-Investigadora. Grupo Dimensiones Humanas

2 CECAR. Colombia. Correo electrónico: blanca.pérez@cecar.edu.co. Magíster en Educación: Sociología de la Educación. Socióloga. Docente-Investigadora. Grupo Dimensiones Humanas. Directora de la línea de investigación Vulnerabilidad Social y Grupos Poblacionales

3 CECAR. Colombia. Correo electrónico: elcie.tamara@cecar.edu.co. Directora de Bienestar Universitario. Magíster en Educación. Trabajadora Social. 
con una población de 192 IA ${ }^{1}$ de la subregión Mojana abordando las variables y categorías mencionadas. Los resultados indican la configuración de un grupo en alto riesgo social con doble vulneración de derechos, por su condición de pobreza y las secuelas de la ola invernal, lo que trasciende en su desarrollo y garantía de bienestar en el marco de la Convención de los derechos de los niños (Naciones Unidas-CDN 1989).

Palabras clave: infancia y adolescencia, ola invernal, condiciones de vida.

\section{INTRODUCCIÓN}

La subregión Mojana, de gran riqueza natural y ventajas comparativas en relación con los altos niveles de pobreza se sitúa en el Caribe Colombiano y está delimitada geográficamente por el Río Cauca al oriente, el Río San Jorge y la ciénaga de Ayapel al occidente, el Brazo de Loba (Río Magdalena) al oriente y la serranía de Ayapel al sur. Abarca los municipios de: Nechi (Antioquia); Magangué, San Jacinto del Cauca y Achí (Bolívar); Ayapel (Córdoba) y Guaranda, Majagual, Sucre, Caimito, San Marcos y San Benito Abad (Sucre). La Mojana es parte de lo que se denomina la depresión Momposina, una amplia área que por recibir la escorrentía de los valles interandinos, se comporta como delta aluvial interior de la tierra baja e inundable en variada intensidad durante ciertas épocas del año, (Plan Departamental de Desarrollo de Sucre, 2012-2015).

La subregión Mojana, se encuentra localizada en el extremo sur del Departamento de Sucre, conformada por los municipios de Sucre, Majagual y Guaranda; siendo la zona más afectada por la Ola Invernal del 2010. El informe precisó que en otros tiempos de invierno las inundaciones se presentaban con una duración de 2 a 3 meses; pero que para 2010 el fenómeno se había presentado con una duración de 7 meses y con niveles de altura nunca antes alcanzados de 2 a 3 metros. Sus pobladores, se vieron grandemente afectados por "los desbordamientos de las aguas de los ríos Cauca, Magdalena y San Jorge causando pérdidas considerables en las familias, las viviendas, el sector agropecuario, la infraestructura educativa, vial y la Red Hospitalaria", (Plan Departamental de Gestión del Riesgo de Sucre, 2011). Agregó, que generó deterioro en la infraestructura habitacional e institucional, desencadenándose un problema de salud pública con el colapso de las pozas sépticas y sistemas de alcantarillado, se presentó carencia de agua potable y alimentos por los daños en los servicios públicos, la pérdida

1 En adelante IA será la sigla utilizada para referirse a la Infancia y adolescencia. 
de cultivos y del bien pecuario. Del informe se infiere que los datos estadísticos hacen hincapié en las pérdidas de bienes materiales y afectaciones en general; pero no se indica acerca del número de IA, y lo que ellas representan para su desarrollo integral. Al mismo tiempo, queda un vacío de conocimiento acerca de la protección que se debe proporcionar a la IA ante situaciones de emergencias naturales, de acuerdo a lo que establece la Ley de Infancia y Adolescencia o ley 1098 (2006).

Como se aprecia en la información anterior, el análisis del riesgo consiste en identificar y evaluar posibles daños y pérdidas como consecuencia del impacto de una amenaza sobre una unidad social en condiciones vulnerables, con el fin de contribuir al desarrollo político, económico, ecológico y social de los países en desarrollo en procesos de reformas para mejorar de forma sostenible las condiciones de vida de la población, (Cooperación Técnica Alemana - GTZ, 2010).

Se planteó como fundamento teórico para el análisis de las variables el enfoque de bienestar y buen vivir desde los postulados de (Sempere, J., Acosta, A., Abdallah, S. \& Orti, M. 2010), a través del cual se "considera que la persona debe ser entendida como un ser multidimensional, a través del cual, se identifica el bienestar como la satisfacción de una serie de "necesidades" objetivas, que traducidas para este trabajo son Salud, Educación, Hábitat y Participación".

De acuerdo con los autores, el bienestar es definido como "un estado dinámico en el cual el individuo puede desarrollar su potencial, construir relaciones sólidas y positivas con otros y contribuir a su comunidad. El modelo incorpora el "sentirse bien" como experiencia de vida, pero también el "encontrarse bien" en términos de funcionar bien y satisfacer necesidades; a la vez, subraya el papel que desempeñan los recursos personales en la capacidad para comprender las problemáticas sociales, vivenciales, culturales, entre otras, que podrían ubicarse a su alrededor.

En definitiva, se pretendió configurar la satisfacción de las necesidades básicas de IA en contraste con la experiencia de la ola invernal y las afectaciones en su modo de vida, en términos de encontrarse bien y sentirse bien en lo personal; aún en medio de situaciones adversas, cuestión que condujo al punto de quiebre de la discusión en este artículo.

\section{MÉTODO}

La investigación fue de corte complementario, basado en el método de análisis cuantitativo y cualitativo de los hechos ocurridos y las acciones humanas con el fin de analizar y comprender mejor la realidad de los IA 
afectados por la ola invernal. Desde lo cuántico se realizó valoración médico - nutricional, se identificaron las características del hábitat, el acceso a la educación mientras que en lo cualitativo se privilegió la narrativa de la IA acerca de la experiencia vivida. El universo lo constituyeron los 73.552 IA menores de 18 años de "La subregión Mojana" y la población fue de 8.620 IA de 10 a 14 años, tomada del Departamento Administrativo Nacional de Estadística - DANE (2005). La muestra estratificada con un nivel de confianza del $95 \%$ y margen de error del $5 \%$ la compusieron 192 IA en edades de 10 a 14 años de los municipios seleccionados; ya que durante el fenómeno tenían 6 años aproximadamente y podían hablar desde su experiencia. Para distribuir la muestra se procedió a extraer los datos porcentuales por municipio para delimitar el tamaño de la misma, así:

\begin{tabular}{|r|c|c|c|}
\hline \multicolumn{4}{|c|}{ TAMANOO DE MUESTRA ESTRATIFICADA PARA ESTIMAR PROPORCIONES } \\
\hline MUNICIPIOS & $\begin{array}{r}\text { POBLACIÓN } \\
\text { AFECTADA }\end{array}$ & $\begin{array}{c}\text { PROPORCIÓN POR } \\
\text { MUNICIPIOS }\end{array}$ & $\begin{array}{c}\text { TAMAÑO DE } \\
\text { MUESTRA }\end{array}$ \\
\hline MAJAGUAL & 3884 & $45,1 \%$ & 86 \\
\hline SUCRE-SUCRE & 2679 & $31.1 \%$ & 60 \\
\hline GUARANDA & 2057 & $23 \%$ & 46 \\
\hline TOTAL & 8620 & $100 \%$ & 192 \\
\hline
\end{tabular}

Fuente: Cálculo realizado por los investigadores del tamaño de la muestra estratificada (2014)

Inicialmente con la Oficina de Riesgo de cada Municipio, se obtuvo información de las zonas más afectadas en cada una de ellos, teniendo en cuenta casco urbano y rural; así: en el municipio de Sucre los más afectados fueron Isla Grande y el Cedro, en Guaranda Palmaritico y Tierra Santa; en Majagual Palomar y Pueblo Nuevo.

Se realizaron mesas de trabajo con los Operadores de la Política Pública en cada una de las cabeceras municipales: Comisarios de Familia, Policía de infancia y adolescencia, funcionarios de Bienestar Familiar, corregidor, inspector de policía en el área rural a fin de obtener un mapeo de las zonas mayormente afectadas y abrir el debate acerca del cumplimiento de los derechos frente a la situación de la IA afectada por la ola invernal en cuanto a las variables salud, Hábitat y educación.

También se aplicó la técnica Grupo Focal a IA de 10 a 14 años, a fin de explorar la categoría de participación de los mismos en la vida familiar, 
escolar y lo relativo a la experiencia vivida durante la ola invernal. Se tomaron muestras de los municipios zona rural y cabecera; para un total de 11 grupos focales.

Finalmente, se realizaron 86 encuestas a padres y/o adultos responsables de la IA que posibilitó constatar sus condiciones de vida y las de sus familias, a partir del análisis de variables educación, salud y hábitat. El procesamiento y tabulación de la información recolectada en encuesta se hizo a través de SPSS 18 y para el análisis de las narrativas de la IA y las entrevistas a funcionarios públicos, se utilizó el programa ATLAS TI. Seguidamente se triangularon los datos para la respectiva interpretación, análisis y discusión.

Este artículo focaliza su interés en el análisis de las condiciones de vida material de la IA examinado derechos fundamentales como son la salud, hábitat, educación y participación de tal modo que se constituya en una provocación para triangular con las variables estado emocional y niveles de adaptación desde lo cognitivo, también examinadas en ésta investigación.

\section{ANÁLISIS Y DISCUSIÓN DE RESULTADOS}

En primera instancia, se exponen las características socio demográficas de la población, a fin de reconocer el entorno de vida de los habitantes de ésta subregión que presenta indicadores de necesidades básicas insatisfechas, es así como el 97,78\% de la población se sitúa en la escala 1 de estratificación social (Ley 142 de 1994) Un 56,67\% de los hogares encuestados habitan en zonas rurales, no obstante, el resto de la población localizada en cabeceras municipales vive en condiciones similares a las del campo, con deficiencias en la infraestructura vial y en los servicios básicos de saneamiento. El sustento de la economía es agrícola en el orden del 52,22\%, es decir devienen del arado de tierra, sembrados, recolección de alimentos, cosechas de cultivos, arroz, yuca, plátano y maíz; con ingresos promedio de menos de $\$ 150.000$ mensual, lo que indica menos de 2 dólares diarios para subsistir con una familia numerosa expuesta a mínimas condiciones de vida, hecho que se agudiza con la experiencia de la ola invernal. El principal proveedor económico es el padre en un 57,78\% encargado del rol instrumental del hogar, es decir de la seguridad económica, alimentaria y de vestuario, mientras que la mujer asume un rol afectivo encargada de la responsabilidad en los quehaceres del hogar y de la educación y crianza de los hijos. Se identificó que la IA en un alto porcentaje convive con sus padres y hermanos, conformándose familias de tipología nuclear y ampliada, en hogares constituidos en unión libre, tanto para la zona rural como para la cabecera municipal. 
El 75.8\% de los hogares rurales de la subregión Mojana, se encuentra en condiciones precarias, con muy altas necesidades básicas insatisfechas, en materia de vivienda inadecuada, de escasa cobertura de saneamiento básico, de inasistencia infantil escolar. Estos indicadores demuestran la situación de rezago socioeconómico histórico de los municipios de La Mojana, con efectos negativos en la población rural en materia de viviendas inadecuadas, hacinamiento crítico, escasez de bienes públicos, como el saneamiento básico, inasistencia escolar, y alta dependencia económica del Jefe del hogar. En el año 2010 y como consecuencia de la ola invernal; emigraron de esta zona 2.704 personas de un total de 3.781 personas expulsadas a los departamentos de Bolívar y Sucre, o sea el 71\%; lo que permite deducir la gravedad de la emergencia, (Oficina de Prevención y Atención de Desastres de Sucre - OPADES, 2012).

\section{Condiciones de salud}

En lo pertinente a la salud, se encuentra que la región Mojana no cuenta con servicio de alcantarillado, el agua no es apta para el consumo humano y el tratamiento de los residuos sólidos no se contempla en el ámbito urbano, mucho menos para el sector rural. Lo anterior, empeora las condiciones de salud de la IA en la época invernal por el consumo de aguas contaminadas de represas, caños y riachuelos, que causaron enfermedades de piel en un $31,11 \%$, enfermedades diarreicas agudas $30 \%$ y un $26 \%$ presentó infecciones respiratorias agudas. En lo que respecta al peso y talla se identificó que el $50.0 \%$ fue bajo según los índices de medición de la escala nutricional siendo este un indicador importante para estimar inadecuada salud nutricional. Es evidente que las circunstancias que la ola invernal trajo consigo, además, de las ya presentes en el contexto fueron un detonante para desmejorar la salud de la IA, si se precisa que la salud nutricional está asociada no solo al conocimiento de los nutrientes de los alimentos, la preparación de éstos y la baja capacidad de compra de alimentos: sino también a factores ambientales que causan desnutrición, enfermedad, retraso del crecimiento, desarrollo psicomotor y disminución del sistema inmunológico, que potencia el contraer infecciones y aumenta el riego de mortalidad, (Organización de las Naciones Unidas para la Agricultura y la Alimentación - FAO, 2014). Todo ello implica un desajuste en el organismo lo que se advierte en el estado de ánimo, el rendimiento escolar y las relaciones familiares y sociales.

El panorama de salud empeoró ante este hecho climático por lo que se decretó la emergencia hospitalaria, especialmente en La Mojana (Decre- 
to 4580 de 2010) debido a las deficiencias en la infraestructura hospitalaria de los centros de salud afectados por el fenómeno vulnerando los derechos de los pacientes a ser atendidos adecuada y oportunamente (Ley 100 de 1993).El 90 \% de la población Mojanera está vinculada al régimen subsidiado, lo que es otra pista para confirmar las condiciones de pobreza en que habitan.

No obstante que el Estado colombiano desde las instituciones del Sistema Nacional de Bienestar Familiar (SNBF) responsables de brindar protección a la IA con el acompañamiento de organizaciones no gubernamentales como la Cruz Roja Internacional, la Defensa Civil realizaron brigadas de salud, entrega de ayudas humanitarias, suministro de medicamentos y albergues provisionales a los afectados en escuelas e Iglesias. El apoyo del sistema general de atención a la población en riesgo fue insuficiente ante las demandas de la población en materia psicosocial para atender a las familias y recuperar la zona afectada. Las condiciones de salud de la IA, siguen siendo críticas, como lo son para todas las poblaciones rurales del Departamento, (Plan municipal de Gestión del Riesgo de Desastres de Guaranda, 2012).

\section{Condiciones de hábitat}

Se presenta una estabilidad residencial de las familias en el casco urbano y rural, lo cual se evidencia en la tenencia de las viviendas, pues un $74,44 \%$ de estas son propias, pero construidas a las orillas de los ríos, arroyos, y expuestas a riesgos propios de la zona geográfica. Otro aspecto a resaltar es la distribución de las viviendas y los espacios con los que cuenta, pues se hallaron viviendas que no tienen baños, dos alcobas para un hogar cuyo tamaño oscila entre 4 a 5 personas, por lo que viven hacinados "comparto cuarto con mi hermana, con la cuñada y la sobrina". GF Majagual. Ante la ola invernal construyeron zarzos y tambos ${ }^{1}$ para asegurar sus enseres y dormir en hamacas dado el alto nivel del agua. No obstante, las familias expresan han recibido apoyo económico y logístico por parte de Colombia Humanitaria, Programa la Mejora, población víctima del desplazamiento, Familias en Acción, entre otros para la reconstrucción de sus viviendas; estas aún no se ejecutan "es que dicen que van a hacer mejoramiento de vivienda y no llega nunca" GF Isla Grande, Sucre.

1 Construcciones hechas en madera para proteger los enceres de la casa de las corrientes de agua producto de la ola invernal. 
Durante la emergencia, se brindó todo el apoyo temporal desde organizaciones gubernamentales y no, para reubicar a las familias en sitios más altos, casas improvisadas, cambuches, pero transcurrido el tiempo las poblaciones vuelven a sus viviendas localizadas a orillas de los ríos; es decir, expuestas a la nueva emergencia y en peores condiciones de vida. Lo que se traduce en intervenciones reactivas por parte del estado y a largo plazo sus condiciones de vida siguen siendo las mismas o peores ante las pérdidas.

En lo que respecta a Vivienda, se tiene que la mayoría no alcanza a cumplir con las condiciones mínimas de vivienda adecuada que estipulada el Comité de Derechos Económicos, Sociales y Culturales - CESCR, pues, son viviendas construidas con materiales que no soportan una situación de peligro frente a una arremetida de ola invernal. De acuerdo a este Comité "las personas como las familias tienen derecho a una vivienda adecuada, independientemente de la edad, la situación económica, la afiliación de grupo o de otra índole, la posición social o de cualquier otro de esos factores. En particular, el disfrute de este derecho no debe estar sujeto, según el párrafo 2 del artículo 2 del Pacto, a ninguna forma de discriminación, (CESCR, 1985).

Se encontró además, que la distribución de las viviendas y los espacios con los que cuenta para garantizar el normal desarrollo de las necesidades en la familia, pues se hallaron viviendas que no tienen baños, además de un número reducido de habitaciones para el número de personas; además de que estas familias para la temporada de ola invernal, duermen en sitios improvisados (zarzos, tambos, hamacas y cambuches) que no aseguran un bienestar general. La importancia de una vivienda digna es un tema de larga data y que aún es vigente en los distintos discursos relacionados con los derechos. La IA no contaba antes de la ola invernal con el confort necesario para su desarrollo y comodidad, mucho menos en la emergencia; cosa que es probable no haber sido identificado o reconocido por los mismos habitantes, no obstante, el crudo invierno deterioró las condiciones precarias de vida.

Estas características relacionadas con el desarrollo integral y con los derechos de los IA no son propias solo de la temporada de ola invernal, lo que ocurre es que se acentúa la desesperanza, pues queda en las mentes de muchos Niños, Niñas y Adolescentes el sin sabor de lo ocurrido en su familia y las limitaciones que se presentaron para garantizarles un mínimo de bienestar que incluyera acceso a todo lo que concierne el desarrollo in- 
tegral. Situación que se agravó dado a que en los gobiernos anteriores "se abandonó por completo el manejo de los riesgos y desastres como concepto preventivo y la acción gubernamental se orientó hacia la atención de las emergencias", (Cárdenas, Camilo., 2012).

\section{Condiciones de Educación}

En cuanto a educación, se evidenciaron bajos niveles de escolaridad y con ello pocas posibilidades de movilidad social en los hogares, pues un $47,78 \%$ de los padres de familia sólo ha cursado primaria, lo que deja entrever que a pesar de los esfuerzos en el desarrollo de programas y proyectos educativos implementados en Colombia para superar los índices de analfabetismo; aún se encuentran padres y madres cabezas de familia iletrados, lo que impide superar las condiciones de pobreza.

La educación para la IA afectada por la ola invernal fue aplazada, dada la emergencia y el estado de alerta que fue declarado para toda la zona ribereña que, pues, algunas de las instituciones educativas fueron utilizadas como sitio de albergue para las familias damnificadas que vivían a orillas de los ríos San Jorge, Magdalena y Cauca, como lo constata el coordinador del sistema de Gestión de Riesgo de la Mojana:

para esta emergencia hubo bastante protección del Estado, la Cruz Roja, la defensa civil, el ejército duplicó sus fuerzas; también hubo ayuda humanitaria en el sentido de alimentación, agua; y la emergencia se superó. Para los albergues se utilizaron las instituciones educativas más que todo la Normal, la iglesia sirvió de albergue y hubo esa atención.

Frente a esta expresión, se infiere que superar este tipo de emergencia solo implica dar lo básico agua, alimentación y posada momentánea; cuando realmente significa volver a la normalidad a la cotidianidad, regresar a la escuela, al hogar y realizar las actividades que se venían desarrollando regularmente, antes por el contrario se rompe la dinámica social, se gestan otros modos de vida que desarticulan las relaciones sociales, entrando en un proceso de incertidumbre que afecta la salud mental. En esta misma expresión se señala abiertamente la afectación en el área escolar, las instituciones educativas se convierten en alojamiento.

Reconfirma esta opinión, la secretaria de Salud de Majagual Sucre:

la educación fue una de las la más afectadas en esta época y la salud, en educación por lo menos fueron cerca de 3 meses sin clases, esto hacia que afectara el rendimiento académico de los niños, las ICFES bajaron a un nivel pésimo durante ese año. Como padre se puede decir que esta fue una de las principales afectaciones, pues muchas familias optaron por llevarse a sus hijos a otros sitios como Sincelejo y allá tienen que colocarle 
refuerzo por que no están al mismo nivel de aprendizaje, ejemplo: niños que cursaban 10 y 11 en los porcentajes de las pruebas ICFES se evidencia la deficiencia y muestra la dificultad para ingresar a una universidad y esto genera un trauma en ellos y por eso si se ve la afectación.

El medio de transporte utilizado para el traslado a las Instituciones Educativas independiente de la temporada de ola invernal es la canoa en un $60 \%$, resaltando la mayor prevalencia durante la emergencia, mientras se suspenden las labores académicas. No obstante, algunos estudiantes se trasladan a pie hacia las instituciones o a los sitios estratégicos dispuestos para vincularse a su proceso educativo.

Para el caso de las cabeceras municipales, se tuvo un periodo de asistencia a clases contando con el traslado de los estudiantes en lanchas y chalupas ${ }^{1}$ hacia los establecimientos educativos, mientras que para el área rural, los Niños, Niñas y Adolescentes demoraron alrededor de 2 meses sin asistir a sus labores académicas, lo cual retardó el proceso educativo incidiendo en el aprendizaje y su rendimiento académico, así como en la motivación del estudiantado para asistir a la escuela, dado que la infraestructura de las instituciones se vio afectada. Al respecto los Niños, Niñas y Adolescentes manifestaban que no les gustaba el ambiente de su escuela por la humedad, los desechos y malos olores con los que quedaban los salones y sus demás espacios, además por las enfermedades que se propagaban en el medio.

Haciendo alusión al contexto del barrio, los Niños, Niñas y Adolescentes referían que también quedaba en situaciones muy deprimentes, puesto que las calles, las vías de acceso y las casas permanecían destruidas, con grietas, malos olores, huecos, basuras y demás que dificultaba las posibilidades de recreación, ocio, de convivencia y de traslado hacia los lugares donde frecuentemente asistían.

En este sentido y dada las dificultades de esparcimiento, los Niños, Niñas y Adolescentes reducían sus espacios de recreación a la sala de sus casas, para prevenir cualquier tipo de situaciones que pusiera en riesgo sus vidas. En tanto, se encontraron Niños, Niñas y Adolescentes a quienes se les permitía salir a jugar en horas del día y con límites de tiempo, haciéndolo dentro del agua. Cabe resaltar que sus juegos preferidos rescatan algunos juegos tradicionales propios de la región como la bolita, el bate, el congelado, el cacho, el futbol, entre otros. En la narrativa de la IA, denota los sentires de la experiencia en sus vidas "No me gusta porque no podemos

1 Medio de transporte acuático utilizado para el traslado entre municipios cercanos 
jugar", "No porque está lleno de agua y no puede venir uno a estudiar como todos los días", "Es muy frío y así hay malas enfermedades".

Los anteriores relatos demuestran claramente el incumplimiento de los derechos a la educación (artículo 67 de la Constitución política de Colombia, 1991) y recreación (artículo 44 de la Constitución política de Colombia, 1991) de la población IA, aspectos estos de suma importancia para el desarrollo integral y obviamente indicadores de condiciones mínimas de vida.

\section{Condiciones de Participación}

En materia de la participación de la IA se encuentra una limitación, pues un $64,44 \%$ no participa de los asuntos familiares ni en los que se relacionan directamente con su bienestar, sin embargo, los padres expresaron que les preguntaban a los niños, niñas y adolescentes, sobre temas relacionados con traslados hacia otros lugares durante la ola invernal. El código de infancia y adolescencia estipula en su artículo 31 que "los niños, las niñas y los adolescentes, tienen derecho a participar en las actividades que se realicen en la familia, las instituciones educativas, las asociaciones, los programas estatales, departamentales, distritales y municipales que sean de su interés". La ley es muy clara, los padres asumen que cumplen como tal, pero las posibilidades de participación para la IA son mínimas en un mundo de limitaciones para los adultos y en consecuencia para los niños como sujetos de derecho.

Cabe resaltar que la participación que se da es entendida como la que tienen en la escuela y la comunidad en concursos, bailes y juegos deportivos. De ninguna manera se concibe como la oportunidad de sentar puntos de vista, opiniones, posiciones frente a sus derechos como sujetos activos. Para los mismos niños, hay desconocimiento de que su voz cuenta.

En cuanto a las experiencias vividas frente a la ola invernal sus expresiones son ambivalentes; mientras que para algunos la ola invernal hace parte del modo de vida al que se han adaptado porque pueden divertirse y compartir con amigos, en donde nadan, pasean en canoa, para otros representó malestar y desagrado.

"...nos sentimos muy mal porque en la ola invernal nosotros no podíamos salir y porque en el agua habían muchas serpientes y muchas cosas peligrosas para nuestra salud" "yo me la pasé bien porque me la pasaba jugando con mis hermanos, nos poníamos a jugar en la calle y todo está anegado" "...bueno, muchas veces se sentía un ambiente triste porque, generalmente cuando se empezaba a inundarse y estábamos en Diciem- 
bre y muchas veces no teníamos para donde salir porque todo estaba inundado y no había espacio" "...me sentía un poco mal pero al mismo tiempo bien porque de pronto a mí me gusta el deporte nadar, pero como pasan meses uno también se aburre y después que ya llegó el seco, o sea el suelo me sentía bien porque ya uno podía otra vez usar zapatos y hacer cosas que no podía hacer cuando estamos en medio del agua".

La infancia y adolescencia opinó que una vez pasó la ola invernal, retomaron nuevamente las actividades cotidianas y aunque muchos espacios se encontraban destruidos o en mal estado, sentían alivio y alegría principalmente para tener la libertad de jugar y recrearse.

"...cuando se acaban las inundaciones, por lo menos sentimos un alivio que ya pasaron esos momentos de dificultad y que por lo menos tenemos un ambiente más sano en que recrearnos". "...me sentí muy bien, ya que cuando se fue la ola invernal nosotros podíamos salir y jugar con nuestros amigos y así preveníamos muchas enfermedades". "... bueno yo me siento feliz, porque así como pasó la inundación del 2010 que inundó diciembre, lastimosamente muchos no pudimos salir. Eso me dio fue tristeza, entonces cuando ya se acabó el agua para mí fue una felicidad porque ya volví a ejercitar los pies jugando futbol".

Cabe mencionar lo planteado por los funcionarios públicos en lo pertinente a las acciones realizadas en la temporada de ola invernal, quienes ostentaron que ante la intención de atender la emergencia, desde la Administración municipal y la oficina de gestión y riesgo de la subregión, se tomaron medidas apoyadas en la Unidad Nacional para la gestión de Riesgo de desastres y el Fondo de Adaptación en cuanto a la asignación de subsidios de sostenimiento que según lo expresado por el Dr. Fidel Serrano, Coordinador de la oficina gestión de riesgo de La Mojana Sucreña, hacia parte del restablecimiento económico en materia de servicios, préstamos y créditos que debían ofrecérsele a toda la población una vez se encontrara censada e incluida en los programa del Fondo de Adaptación.

En cuanto a la identificación de la población mayormente afectada por la ola invernal 2010, estos manifestaban que no se encuentran datos estadísticos por distribución etaria, ni reportes de las zonas y poblaciones con mayor vulnerabilidad, en este sentido, los funcionarios desconocen las cifras de población infantil y adolescente afectada y el referente que manejan es el dato poblacional de afectados por núcleos familiares de las diferentes zonas, información que soporta el Dr. Jesús Taborda Secretario de Salud del municipio de Sucre - Sucre, ubicado en la región Mojana "No hay esos datos estadísticos, es más, yo creo que ni el mismo DANE sabe porque globaliza por familia, y nosotros como instituciones municipales tampoco nos 
damos la tarea de hacer esos datos estadísticos".

Se hace un llamado a todos los actores locales y sociedad civil en general para configurar escenarios de participación en los que la IA pueda ser escuchada como sujeto social de derecho y autogestionarios de su propio desarrollo, pues, existe una gran limitante en cuanto a oportunidades de participación y toma de decisiones frente a las problemáticas que los aquejan. Como derecho humano la participación es un derecho civil al que se tiene acceso en el ámbito social, por lo tanto, debe ser promovida y aceptada, pues debe potenciarse en este grupo poblacional, las capacidades para comprender y reconocerse en su propia realidad. Se puede inferir que existe un grado de afectación en cuanto al cumplimiento de derechos de esta población, pues, aunque legalmente se ha estipulado la obligatoriedad en la garantía de una vida digna y el goce efectivo de los derechos, no se asegura salud, vivienda, educación y participación que propenda por garantizar un estado de completo bienestar para la plena satisfacción de una serie de "necesidades" a nivel individual y colectivo de esta población que ha experimentado la ola invernal, ya que se genera un aplazamiento en el acceso a mejores condiciones de vida que garanticen un bienestar integral.

En las opiniones de los Niños, Niñas y Adolescentes sobre el sentirse bien en las experiencias de vida frente a los cambios climáticos, consideraron por una parte la afectación en términos de cambio de vida y de acceso a la educación, vivienda, salud y participación; y para otros la ola invernal fue un modo de vida al cual se habían adaptado.

Para finalizar, en materia de la conceptualización de Sempere, J., Acosta, A., Abdallah, S., \& Orti, M. (2010), que se referenció anteriormente, respecto al bienestar de las personas a través de la satisfacción de las necesidades, amerita una profunda reflexión puesto que el grupo de IA no alcanzó de acuerdo al modelo propuesto por ellos ni el sentirse bien ni tampoco encontrarse bien, las carencias fueron demasiado marcadas para posibilitar un ser y estar adecuado, aun cuando en el momento algunos de ellos, por su edad no visibilizaron la magnitud del problema, manifestando sentirse bien, pero a mediano y largo plazo se fueron estructurando otros sentires y estados de ánimo que denotaron que el bienestar estaba lejos de obtenerse. El buen vivir, entonces, tiene una trascendencia mayor a la sola satisfacción de necesidades y acceso a servicios y bienes, implica adaptación, bienestar emocional, espiritual y psicológico.

Este trabajo investigativo fue transcendental, puesto que el conocimiento de las condiciones de vida y de las significaciones dadas al suceso 
por parte de la población IA, posibilitó diseñar estrategias de promoción y prevención frente al riesgo ambiental. Además de altos niveles de insatisfacción básicos; la IA junto a su familia, carecía de información ilustrativa que les permitiera educarse frente al riesgo. En consecuencia, el equipo investigador aportó el diseño y creación de un juego didáctico llamado ACUAKID, es un juego de mesa en el que participan seis jugadores los que con el uso de un dado intentan sortear obstáculos para lograr la meta y en su recorrido encontraran preguntas y respuestas acerca de las buenas y malas prácticas frente al riesgo ambiental; su propósito es que ID aprendan a identificar los riesgo generados por el cambio climático y buscar posible soluciones a las situaciones vividas.

\section{CONCLUSIONES}

La población afectada por la ola invernal en La Mojana Sucreña desde el 2010, vive en condiciones mínimas con necesidades básicas insatisfechas, en el nivel 1 de la escala de estratificación social, con bajos niveles educativos e ingresos mínimos. Es una población con características similares en el modo de vida, tanto para cabecera municipal como para el resto, dada la ruralidad de la subregión y el desarrollo de actividades para el sostenimiento familiar enmarcadas en actividades agrícolas y del campo.

Las inundaciones ubican a la población infantil y adolescente en un grupo en alto riesgo social por la doble vulneración de sus derechos, dadas sus condiciones de pobreza y las consecuencias de la ola invernal en sus vidas; que limitan el desarrollo integral y la garantía de sus derechos como lo manda la carta convencional. Esta vulneración de derechos se presenta no sólo por la temporada de fuertes lluvias e inundaciones, sino por las condiciones en que viven Niños, Niñas y Adolescentes en un contexto de necesidades básicas insatisfechas, condiciones mínimas habitacionales, lo que propicia la aparición y propagación de enfermedades insuficiente acceso a servicios de salud en el sector rural, poco acceso a la educación una vez se presenta la ola invernal.

Para el Estado Colombiano y la implementación de Política Pública significa la posibilidad de incluir en los Planes de desarrollo Departamental y local, la atención a la población infantil y adolescente que enfrenta situaciones de alto riesgo como el fenómeno de la Ola Invernal en Colombia. La garantía de los derechos de los niños es un ejercicio aplazado por la falta de compromiso de los entes territoriales que condicionan su aplicación según la interpretación y lectura que hacen de las condiciones socioeconómicas de las poblaciones y que influyen directamente en el pleno desarrollo de la 
Infancia y la Adolescencia.

Los operadores de la política pública municipal manifestaron que para la atención e intervención de la población infantil y adolescente afectada por la ola invernal, no figuran programas específicos que les permita atender de manera directa a este grupo poblacional. La atención a la población infantil y adolescente en situación de vulnerabilidad por el cambio climático, es un tema que se ha invisibilizado en el marco de la implementación de políticas públicas a nivel Nacional, Departamental y local; en tanto el sistema de gestión de riesgo no explicita atención prioritaria e este grupo poblacional.

Es de gran importancia recordar que los Niños, Niñas y Adolescentes también hacen parte del núcleo familiar y que, ante ello, debe garantizársele el derecho a opinar, elegir, decidir lo mejor para ellos y en consecuencia forjar el cumpliendo de sus derechos en materia de bienestar y desarrollo integral, lo cual se evidencia y contrasta con la validación desde sus propias experiencias, vivencias, sentir y opinión de este grupo poblacional. Aunque la investigación permitió conocer la población mayormente afectada por la ola invernal en términos generales y sus condiciones de vida; los datos de infancia y adolescencia son imposibles de cuantificar para toda la subregión, pues, los reportes oficiales de afectados hablan de familias, hogares y afectaciones materiales, mas no se registran datos por grupos etarios.

\section{REFERENCIAS BIBLIOGRÁFICAS}

Cárdenas Giraldo, Camilo (2012). La Ola Invernal: Por qué daños y cómo prevenirlos. Recuperado de: http://www.razonpublica.com/index.php/econom-y-sociedad-temas-29/2356-la-ola-invernal-por-que-los-danos-y-como-prevenirlos-. html

Comité de Derechos Económicos, Sociales y Culturales - (CESCR), (1985). El derecho a una vivienda adecuada, art. 11, párr. 1. En: http://www.acnur.org/t3/fileadmin/Documentos/BDL/2005/3594.pdf?view=1

Constitución política de Colombia 1991: art. 67 y 44. Recuperado de: http://www. alcaldiabogota.gov.co/sisjur/normas/Norma1.jsp?i=4125

Cooperación Técnica Alemana - GTZ, 2010: En: http://www.gtz.de/en/weltweit/lateinamerika-karibik/636.htm

Departamento Administrativo Nacional de Estadística - DANE (2005). Censo Nacional.

Decreto 4580 de 2010. Por el cual se declara el estado de emergencia económica, social y ecológica por razón de grave calamidad pública. Recuperado de: http://www.alcaldiabogota.gov.co/sisjur/normas/Norma1.jsp?i=40910

Departamento Administrativo Nacional de Estadística (DANE: 2010-2011) Encuesta sobre Familias afectadas por cambios climáticos. 
Condiciones de vida de la infancia y adolescencia afectada por eventos climáticos del 2010 en La Mojana Sucreña

Ley 142 de 1.994 Ministerios de Salud y Protección Social. Régimen subsidiado. Recuperado de: http://www.minsalud.gov.co/salud/Paginas/R\%C3\%A9gimenSubsidiado.aspx

Ley 142 de 1994. Nivel nacional. Por la cual se establece el régimen de los servicios públicos domiciliarios y se dictan otras disposiciones. Recuperado de: http://www.alcaldiabogota.gov.co/sisjur/normas/Norma1.jsp?i=2752

Ley 1098 de 2006 (noviembre 8). Diario oficial no. 46.446 de 8 de noviembre de 2006. Recuperado de: http://www.secretariasenado.gov.co/senado/basedoc/ ley/2006/ley_1098_2006.html

LEY 100 DE 1993. Recuperado de: http://www.alcaldiabogota.gov.co/sisjur/normas/ Norma1.jsp?i=5248

Naciones Unidas-CDN (1989). Convención sobre los Derechos del Niño (Resolución 44/25, Asamblea General de la ONU, 20 de noviembre de 1989 Aprobada por la ley 12 de 1991.UNICEF. Edit. Nueva.

Oficina de Prevención y Atención de Desastres (OPADES). Reporte de las familias afectadas en Sucre por cambios climáticos para 2012.

Organización de las Naciones Unidas para la Agricultura y la Alimentación - FAO, 2014

Plan de Departamental de Desarrollo de Sucre, 2012-2015. Recuperado de: http:// www.observatoriodeldeporte.gov.co/docs/col/56/attach/plan_de_desarroIlo_sucre_2012-2015.pdf

Plan Departamental de Gestión del Riesgo de Sucre, (2011). División Político Administrativa del Departamento de Sucre por Subregiones. La sub región Mojana. Pág. 19.

Plan Municipal de Gestión del Riesgo de Guaranda, (2012). Riesgos por inundaciones lentas en la subregión de La Mojana Sucreña.

Sempere, J., Acosta, A., Abdallah, S. \& Orti, M. (2010). Dossier: Enfoque de bienestar y buen vivir desde los postulados Recuperado de: https://www.fuhem. es/media/cdv/file/biblioteca/Dossier/Dossier_Enfoques_sobre_bienestar_y_buen_vivir.pdf

_Sempere, J., Acosta, A., Abdallah, S. \& Orti, M. (2010). Dossier: Enfoque de bienestar y buen vivir desde los postulados Recuperado de: https://www.fuhem. es/media/cdv/file/biblioteca/Dossier/Dossier_Enfoques_sobre_bienestar_y_buen_vivir.pdf 